\title{
THE EFFECT OF KNEE POSTURES AND CUSHIONS IN THE LOAD TRANSMISSION OF IMPACT LOADING - AN IN VITRO BIOMECHANICAL PORCINE MODEL
}

\author{
JaW-Lin WANG, YEN-Lin LeE \\ Institute of Biomedical Engineering, College of Medicine and College of Engineering \\ National Taiwan University, Taiwan
}

\begin{abstract}
Degenerative osteoarthritis is the consequence of impact force applied to articular cartilage that results in surface fissuring. Soft cushions and flexed posture are two important factors to reduce the impact force; however, no quantitative information of how soft should the cushion be to prevent the injury and the mechanism of force attenuation of knee joint at neutral and flexed posture was not well documented yet. The objective of current study is hence to find the quantitative shock attenuation of knee joint using different stiffness of cushions when the knee is at neutral posture and flexed posture. A "drop-tower type" impact apparatus was used for testing. Nineteen fresh porcine knee joints were divided into two posture groups, i.e. neutral and flexed posture. All specimens were tested using stiff, medium, and soft cushions. The axial reaction force, anteroposterior shear force, and flexion bending moment were recorded for analysis. We found the flexed posture decreased the axial reaction force and anterior shear force but increased the flexion bending moment. The effect of stiffness of cushions on the mechanical response of knee joint during impact loading was significant for neutral posture but not for flexed posture.
\end{abstract}

Biomed Eng Appl Basis Comm, 2004(August); 16: 165-172.

Keywords: Biomechanics, Knee Joint, Impact Mechanics

\section{INTRODUCTION}

The long-term outcome of repetitive impact loading applied on the knee joint results in the cartilage degenerations. Strategies that reduce the impact loading of knee joints include the decrease of the stiffness of knee joint complex and the change of the knee posture from neutral posture to flexed posture. Both the greater knee flexion angle and lower initial

Received: Jan 12, 2004; Accepted: June 15, 2004

Correspondence: Jaw-Lin Wang, Professor

Institute of Biomedical Engineering, College of Medicine and College of Engineering, National Taiwan University, 1, Sec. 1, Jen-Ai Road, Taipei 100, Taiwan E-mail: jlwang@ntu.edu.tw leg stiffness results in the lower impact force [1]. Nonetheless, Lafortune et al. tested 21 human subjects and found that the softer cushion between the foot and floor is more likely to protect the lower limb system against impact loading than the knee angle strategies [2-3]. The human knee joint are in the order of 20 degree flexed [4] following the foot strike during walking and 30 degree flexed [5] during running. The flexed posture during activities would decrease the reaction force; however, the human body has little chance to control the knee posture during the initial impact phase [1]. The softer cushions would reduce the impact reaction force; nevertheless, there is no quantitative information of how soft should the cushion be to prevent the injury, and the mechanism of force attenuation of knee joint at neutral and flexed posture was not well reported yet. The objective of current study is hence to find the quantitative shock 
attenuation of axial force, anterior shear force and bending moment of knee joint using different stiffness of cushions when the knee is at neutral (straight standing) posture or flexed posture.

\section{MATERIAL AND METHOD}

\subsection{Specimen Preparation}

Nineteen fresh-frozen knee joints from mature swine weighted from $100 \mathrm{~kg}$ to $120 \mathrm{~kg}$ were used in the experiment. The size of porcine knee joint was close to the size of adult human knee joint. The mediolateral width and anteroposterior length of tibia plateau of eight tested porcine specimens were $72.2 \pm$ 2.2 and $41.27 \pm 2.2 \mathrm{~mm}$, and that of human specimen were $67.1 \pm 0.2$ and $41.0 \pm 0.7 \mathrm{~mm}[6]$. The bone mineral density (BMD) of 92 and $120 \mathrm{~kg}$ porcine back legs ranged from 1.053 to $1.183 \mathrm{~g} / \mathrm{cm}^{2}$ [7], which was close to the quality of distal femur of 14 to 16 years old Caucasian girls[8]. In preparation for the testing, the specimens were stripped of all soft tissue except at the knee joint, where all ligamentous, capsular, and intracapsular structures were preserved. For better alignment, the joint complexes were fixed with external fixators first. Two groups of knee posture were defined and prepared. One was the neutral posture group, and another one was the 10 degree flexed posture group. It should be noted that the neutral posture was defined as the fully extension of knee joint; however, there was still a flexed angle from tibia to femur, i.e. 160 degree for the porcine knee joint. The real flexed angle of the 10 degree flexed posture group from tibia to femur was hence around 150 degrees. The specimens were potted with quick setting epoxy (Polyesterputty, Gou-Fon, Inc., Taipei, Taiwan) at both ends after the knee posture was aligned.

\subsection{Experimental Apparatus}

A "drop-tower type" impact testing apparatus was used for the testing (Figure 1). An impactor guided by two rods was dropped from the top of the tower to produce the impact energy. The energy was transmitted to the specimen through the impounder. The cushion was mounted on the top of the impounder. The fixed frame, which was fixed to the guiding rode, was used to align the vertical movement of impounder. The cushion was placed on the top of the impounder to control the impact contact period. The stiffness of designed stiff, medium, and soft cushions that made of plastic rubber and silicon rubber, were $800,180,50$ $\mathrm{kN} / \mathrm{m}$ while the loading speed was $1.4 \mathrm{~m} / \mathrm{sec}$. These three cushions were able to give, approximately, the contact time of impact period between impounder and impactor at levels of 20,40 and 60 mini-seconds when testing a standard rubber bar specimen (stiffness = $1000 \mathrm{kN} / \mathrm{m}$, length $=110 \mathrm{~mm}$ ) at $12 \mathrm{~kg}$ impact mass and $50 \mathrm{~mm}$ impact height (6 J impact enegy) [9]. To give a better feeling of how soft the cushions were, the stiff cushions can hardly be deformed by hand force, while the soft cushions can be deformed easily by finger force. The specimen was mounted vertically below the impounder and above the six-axial force load cell (AMTI MC6-6-4000, Advanced Mechanical Technology, Inc., Watertown, MA, USA). Signals of three dimensional reaction forces and moments from the six-axial force load cell were all recorded at $10 \mathrm{kHz}$ sampling frequency. The signals were then low pass filtered at $1 \mathrm{kHz}$ frequency using Butterworth filtering algorithm.

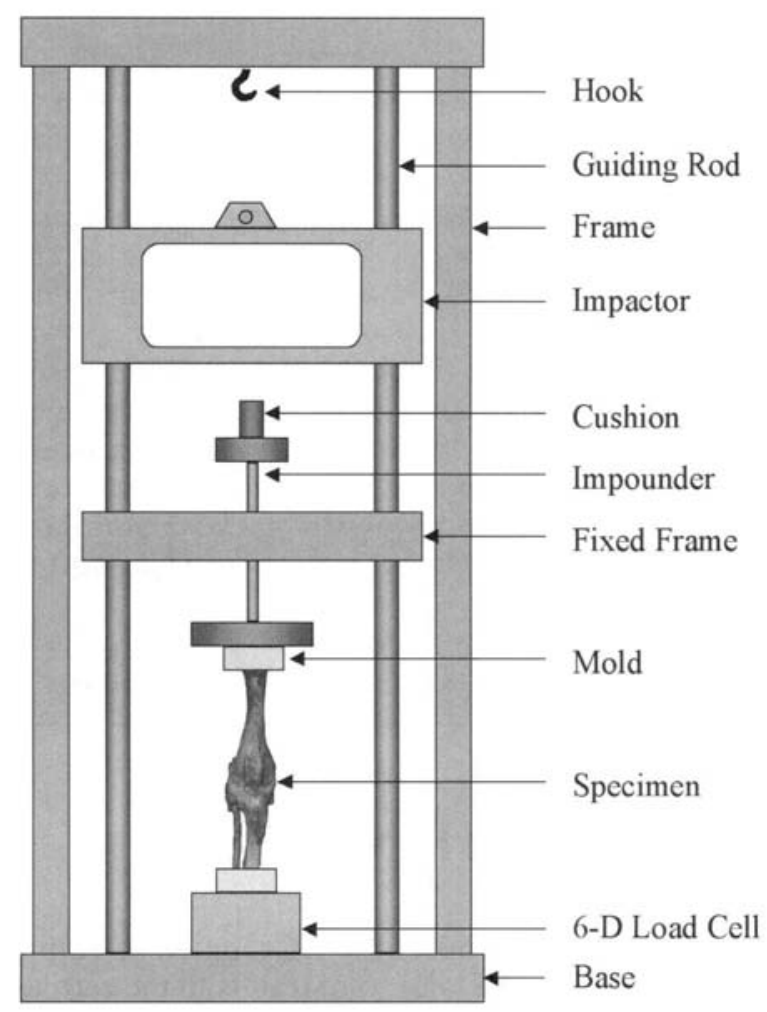

Fig 1. An impactor guided with two rods was dropped from the top of the tower to produce the impact energy. The energy was transmitted to the specimen through the impounder. The cushion was mounted on the top of the impounder. The fixed frame, which was fixed to the guiding rode, was used to align the vertical movement of impounder. The knee joint specimen was placed below the impounder and above the six-axial load cell. The three dimensional force and moment responses were recorded during impact. 


\subsection{Experimental Protocol}

The specimens were divided into two groups, i.e. the neutral posture $(n=10)$ and flexed posture $(n=9)$. All specimens were tested at 30 and $40 \mathrm{~mm}$ levels of height, and at 12 and $16 \mathrm{~kg}$ levels of weight, using three different cushions. The energy input was calculated at the levels of $3.6 \mathrm{~J}, 4.8 \mathrm{~J}$ and $6.4 \mathrm{~J}$. Full factorial experimental design was used. Each specimen was tested for 12 times and 228 experiments in total were conducted. Ten minutes rest, at least, was taken between each impact. The total experimental time for one specimen was finished in four hours to minimize the chronicle variation of mechanical strength of knee joint. The loading history including the axial reaction force, anteroposterior shear force, and flexion bending moment were recorded via a computer. The maximum axial force, anterior shear force and flexion bending moment and the impact contact time were extracted from the loading history (Figure 3).

\subsection{Statistical Analysis}

The responses (dependent variables) of the current study included maximum axial reaction force, anterior shear force, and flexion bending moment. The effects of two factors, i.e. the stiffness of cushions and the knee posture, on the responses were analyzed. The stiffness of cushions was a three levels factor (stiff, medium and soft), while the knee posture was a two levels factor (neutral and flexed). Since both factors were know to be an effective predictor on the mechanical responses of knee joint during impact loading, the multi-factorial ANOVA on the effects of independent factors on the mechanical response was not necessary in the current study. The student t-test was conducted to compare the difference of posture from neutral to flexed at three different stiffness of cushions. We also tried to find the decreased percentages of mechanical responses in three comparisons, i.e. from stiff cushion to medium cushion, from medium cushions to soft cushion, and from stiff cushion to soft cushion for both neutral and flexed posture groups. The changes and its significances were obtained at $95 \%$ confidence level. The statistical software SPSS R10 (SPSS Inc., USA) was used for the statistical analysis.

\subsection{Mathematical Model}

The following mathematical model was used to find the relationship of the mechanical responses with respect to the impact contact time.

$$
Y=\beta+(\alpha-\beta) e^{-t \tau}
$$

The least-squared regression method was use to find the constants $\alpha, \beta$ and $\tau$ with the constraint that $\beta$ must be greater or equal to zero. The correlation of coefficient between experimental data and model was also calculated to find the goodness of fit of the current model. The magnitude of $\alpha$ represents the physical status of variables at time zero, while the magnitude of $\beta$ represents the physical status of variables at steady state. When the impact contact time increased to the value of time constant $\tau$, the magnitude of variables will decrease to $63.2 \%$ of initial magnitude $\alpha-\beta$ (Figure 2). The Matlab optimization toolbox (MATLAB 5.2, Mathworks, Inc., Natick, MA, USA) was used to find the coefficient of the regression equations and the correlation coefficient.

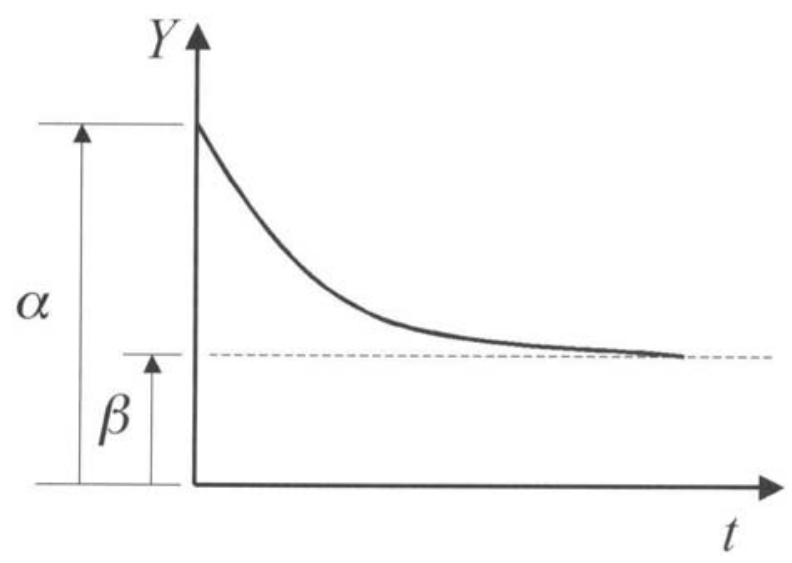

Fig 2. The schematic plot of the regression equation. The magnitude of $\alpha$ represents the physical status of variables at time zero, while the magnitude of $\beta$ represents the physical status of variables at steady state. When the impact contact time increased to the value of time constant $\tau$, the magnitude of variables decreased to $63.2 \%$ of initial magnitude $\alpha-\beta$.

\section{RESULTS}

\subsection{A Typical Impact Pattern}

All specimens were remained intact, i.e. no observable bony or cartilage fracture through out the experiment by visual examination. A typical axial reaction force, shear force and bending moment loading history of neutral standing knee joint during impact loading using three different stiffness of cushions were shown in Figure 3 (specimen \#1, impact mass $=12 \mathrm{~kg}$, impact height $=30 \mathrm{~mm}$ ). The axial reaction force showed a nearly symmetrical haversine. 
while the anteroposterior shear force showed a full sine-wave curve within one impact period. The bending moment was in flexion mode during most of the loading, but with a minor extension in the beginning and the end of the loading. The shear force was in the posterior direction first, and almost invariant with respect to the loading rate, the force reversed to anterior direction at about 7 th $\mathrm{ms}$ after impact. In the current case, the softer cushion decreased the maximum axial forces and bending moment, and increased the time to peak force and moment. The softer cushion also decreased the maximum anterior shear force, but not increased the time to peak force.

\subsection{Statistical Analysis}

The averaged maximum axial reaction force, anterior shear force, and flexion moment reached 2200 $\mathrm{N}, 255 \mathrm{~N}$ and $88 \mathrm{Nm}$ in the sagittal plane when using the stiffest cushion (Figure 4). The axial reaction force was significant higher at neutral posture in comparison with the flexed posture when using the stiff $(p<0.001)$ and medium $(\mathrm{p}<0.001)$ cushions although the variation was not significant when using soft cushion $(p=0.017)$ (Figure 4A). The anterior shear force was significant higher at neutral posture in comparison with the flexed posture when using the stiff $(p<0.001)$ cushion but the variation was not significant when using medium $(p=0.902)$ and soft $(p=0.450)$ cushions (Figure 4B). Nevertheless, the flexion bending moment was significant lower at neutral posture in comparison with the flexed posture when using all three different stiffness of cushions ( $p<0.001$ ) (Figure $4 C$ ).

All the forces and moment decreased with softer cushion. The axial force, anterior shear force and flexion moment of neutral posture knee joints decreased $33 \%, 68 \%$ and $53 \%$ from stiff to medium cushion, respectively. The soft cushion further absorbed the forces and bending moments; however, all decreases were under $20 \%$ (Table 1 ). The effect of cushions on the flexed posture knee joints was a little bit complex. The changes of axial forces among cushions were not significant. The anterior shear force was greatly reduced from stiff to medium cushions $(49 \%, p<0.001)$, but not from medium to soft cushions $(17 \%, p=0.322)$. The changes of flexion bending moment can only be significantly observed from stiff to soft cushions $(42 \%, p=0.007)$, but not from stiff to medium cushions $(15 \%, \mathrm{p}=0.506)$ nor medium to soft cushions $(27 \%, p=0.111)$

It is concluded that the medium cushion, i.e. cushions with 40 mini seconds contact time, can greatly and efficiently absorb the forces and moment of knee joint during impact for knee joints in neutral posture; however, the same conclusion cannot applied to knee joints in flexed posture.
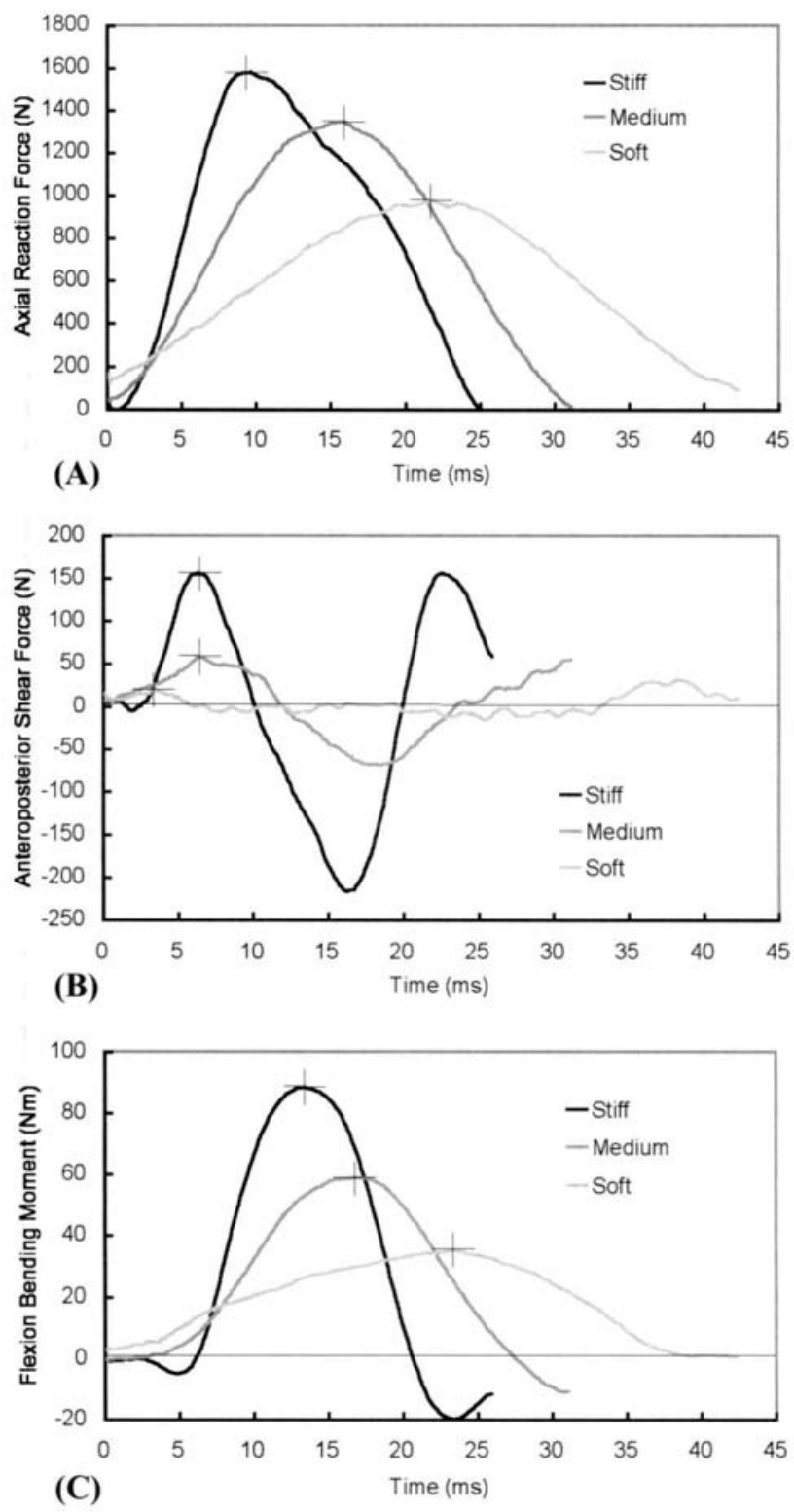

Fig 3. A typical impact behavior of (A) axial reaction force, (B) anteroposterior shear forces and (C) flexion bending moment of neutral standing knee joint (Specimen\#1) was plotted at three loading rate. The loading condition for the current case was $12 \mathrm{~kg}$ impact mass with $30 \mathrm{~mm}$ of impact height. The magnitude of energy input was $3.6 \mathrm{~J}$.

\subsection{Mathematical Regression}

The contact time of neutral posture group ranged from 15 to $55 \mathrm{~ms}$, while the flexed posture group ranged from 25 to $75 \mathrm{~ms}$. The longer impact contact time of flexed posture group remove the knee joint from the risk of high impact force within 15 to $25 \mathrm{~ms}$. 
Table 1. The average ( \pm standard deviation) of maximum value of dependent variables at three different stiffness levels of cushion, and the decreased percentage of dependent variables from stiff to medium cushion, and from medium to soft cushion. One-way analysis of variance (ANOVA) was tested to determine the differences between the stiff and medium cushion, and between the medium and soft cushion.

\begin{tabular}{lcccc}
\hline & & Neutral & Flexed \\
\hline & Comparison & Decreased Percentages & Comparison & Decreased Percentages \\
\hline \multirow{2}{*}{ Axial Reaction } & Stiff-Medium & $33 \%, \mathrm{P}<0.001$ & Stiff-Medium & $-5 \%, \mathrm{P}=0.754$ \\
Force & Medium-Soft & $11 \%, \mathrm{P}=0.026$ & Medium-Soft & $13 \%, \mathrm{P}=0.082$ \\
& Stiff-Soft & $44 \%, \mathrm{P}<0.001$ & Stiff-Soft & $8 \%, \mathrm{P}=0.357$ \\
\hline \multirow{2}{*}{ Anterior Shear } & Stiff-Medium & $68 \%, \mathrm{P}<0.001$ & Stiff-Medium & $49 \%, \mathrm{P}<0.000$ \\
Force & Medium-Soft & $15 \%, \mathrm{P}=0.026$ & Medium-Soft & $17 \%, \mathrm{P}=0.322$ \\
& Stiff-Soft & $83 \%, \mathrm{P}<0.001$ & Stiff-Soft & $66 \%, \mathrm{P}<0.000$ \\
\hline \multirow{2}{*}{ Flexion Bending } & Stiff-Medium & $53 \%, \mathrm{P}<0.001$ & Stiff-Medium & $15 \%, \mathrm{P}=0.506$ \\
Moment & Medium-Soft & $20 \%, \mathrm{P}=0.157$ & Medium-Soft & $27 \%, \mathrm{P}=0.111$ \\
& Stiff-Soft & $73 \%, \mathrm{P}<0.001$ & Stiff-Soft & $42 \%, \mathrm{P}=0.007$ \\
\hline
\end{tabular}

Stiff-Medium $=\left(\mathrm{V}_{\text {Stiff }}-\mathrm{V}_{\text {Medium }}\right) / \mathrm{V}_{\text {Stiff }} * 100 \%$,

Medium-Soft $=\left(\mathrm{V}_{\text {Medium }}-\mathrm{V}_{\text {Suf }}\right) / \mathrm{V}_{\text {Stiff }} * 100 \%$,

Stiff-Soft $=\left(\mathrm{V}_{\text {Stiff }} \mathrm{V}_{\text {Soff }}\right) / \mathrm{V}_{\text {Stiff }} * 100 \%$

The axial force, shear force and bending moment decreased exponentially with the impact contact time for neutral posture knee joint. The axial force and anterior shear force also decreased with impact contact time when the knee joint was in flexed posture; however, the bending moment increased with the impact contact time (Figures 5). The steady state of axial reaction force and bending moment were $1,240 \mathrm{~N}$ and $11.7 \mathrm{Nm}$, while the shear force reached zero if the knee joint was in neutral posture. For flexed knee joints, the steady state reaction force decreased to 725 $\mathrm{N}$, and the shear force and bending moment increased to $70 \mathrm{~N}$ and $136 \mathrm{Nm}$ (Table 2).

\section{DISCUSSIONS}

The in vitro animal study neglected the damping effect provided by the muscles and heel fat pad. The results of current study may represent the worst scenario of impact phenomenon of knee joint during impact loading. The size of porcine knee joint of the current study was close to the human adult. However, the full extension posture of porcine knee joint was still slightly flexed (5 to 10 degrees). This may indicate that the human knee joint may experience higher axial force but lower anterior force and flexion moment during impact loading condition.

The input energy of current study ranges from 3.6 $\mathrm{J}$ to $6.4 \mathrm{~J}$, and the averaged energy was $5 \mathrm{~J}$. Comparing to in vitro tibiofemoral joint impact studies, our energy input was lower than two studies but higher than one (Table 3 ). The maximum axial force reached $3,500 \mathrm{~N}$ at level of $5 \mathrm{~J}$ energy input. Assuming the contact area of porcine knee joint to be $1000 \mathrm{~mm}^{2}$ [11-12], the stress at the cartilage surface was hence $3.5 \mathrm{MPa}$. In the literature, the estimated physiological contact stress of joints of lower extremity was from 5 to $10 \mathrm{MPa}$ [13-14], and the stress threshold of articular cartilage was about $15-20 \mathrm{MPa}$ [15]. The contact stress produced in the current study was well below the injury thresholds of knee injury.

Table 3 insert here

Lafortune et al. [2-3] found that the softer cushion between the foot and floor was more likely to protect the lower limb system against impact loading than the knee angle strategies. In this study, we found that the stiffness of cushions was not important for flexed knee

Table 2. The magnitude of constants $\alpha, \beta$ and $\tau$ and coefficient of correlations of the optimized equations of mechanical response of knee joint at neutral and flexed postures.

\begin{tabular}{ccccc}
\hline Neutral & $\alpha$ & $\beta$ & $\tau(\mathrm{ms})$ & $\mathrm{r}^{2}$ \\
\hline $\mathrm{F}_{\text {Axial }}(\mathrm{N})$ & 17,685 & 1,240 & 7.3 & $80 \%$ \\
\hline $\mathrm{F}_{\text {Ant }}(\mathrm{N})$ & 661 & 0 & 18.0 & $60 \%$ \\
\hline $\mathrm{M}_{\text {Flex }}(\mathrm{Nm})$ & 194 & 11.7 & 19.0 & $43 \%$ \\
\hline Flexed & $\alpha$ & $\beta$ & $\tau(\mathrm{ms})$ & $\mathrm{r}^{2}$ \\
\hline $\mathrm{F}_{\text {Axial }}(\mathrm{N})$ & 2,260 & 725 & 33.7 & $53 \%$ \\
\hline $\mathrm{F}_{\text {Ant }}(\mathrm{N})$ & 2,091 & 70 & 7.9 & $33 \%$ \\
\hline $\mathrm{M}_{\text {Flex }}(\mathrm{Nm})$ & 52 & 136 & 59.7 & $13 \%$ \\
\hline
\end{tabular}




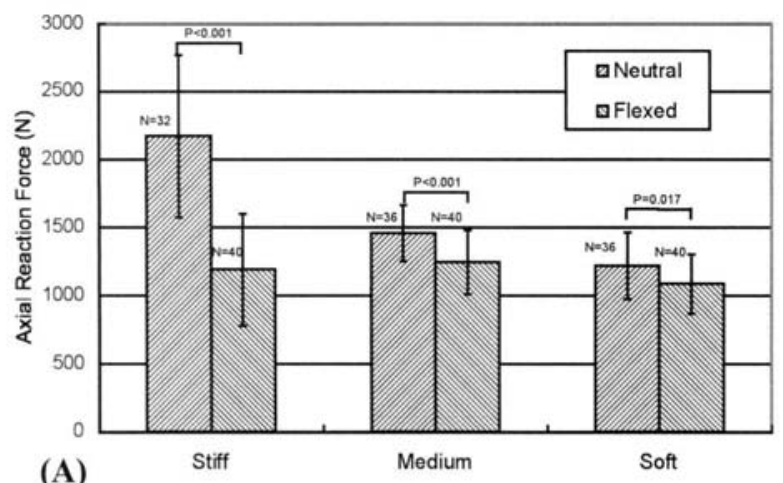

(A)

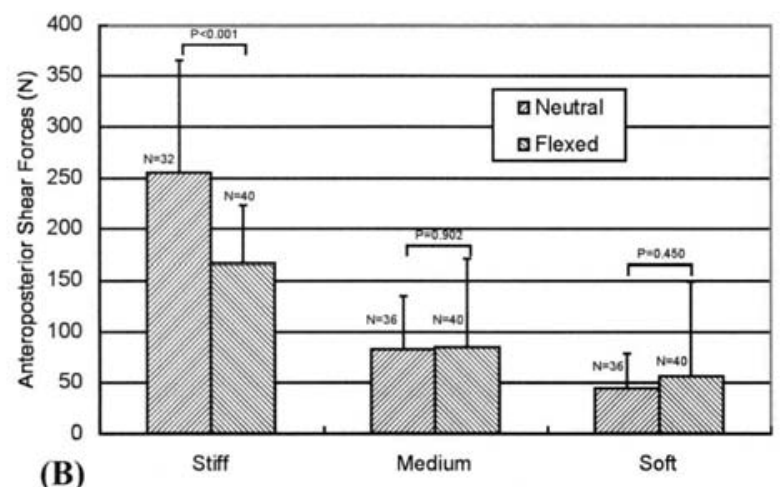

(B)

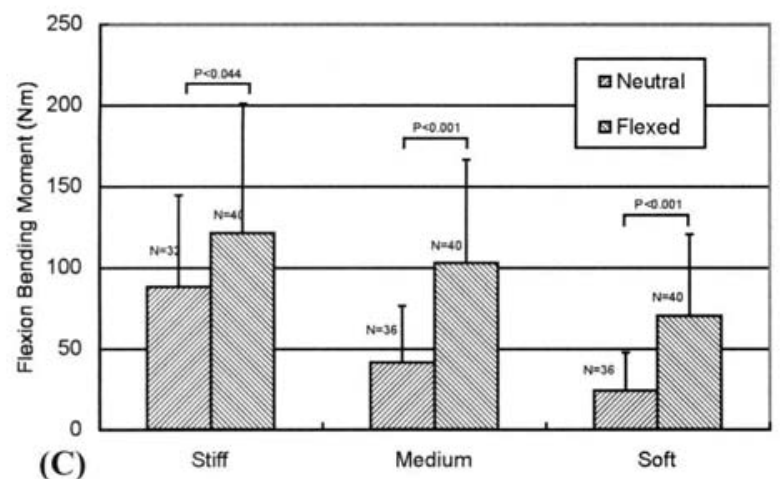

Fig 4. The (A) axial reaction force, (B) anterior shear forces and $(C)$ flexion bending moment of knee joint during neutral (straight standing) posture and flexed posture using three different stiffness of cushions.

joint. In addition, we also showed the soft cushion already greatly reduce the contact force of knee joint. For situation that the knee strategy was not an option, e.g. distance jumping, the only way to reduce the contact stress was to find a proper stiffness cushion. Nevertheless, The effect of knee angle strategies and the confounding factor between these two variables was worth of studying in the future.

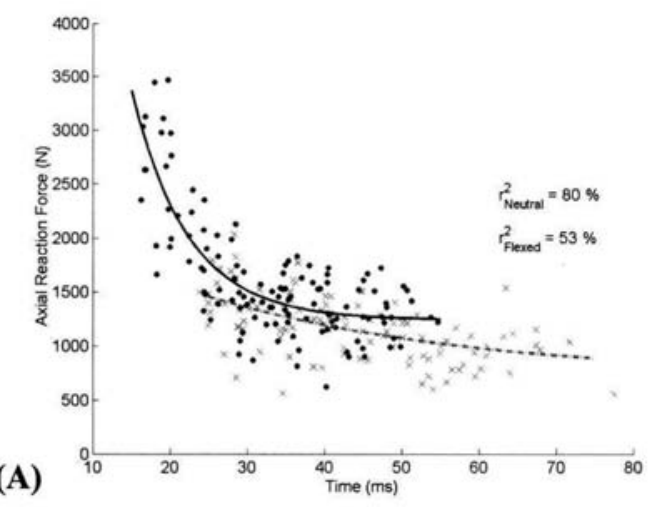

(B)
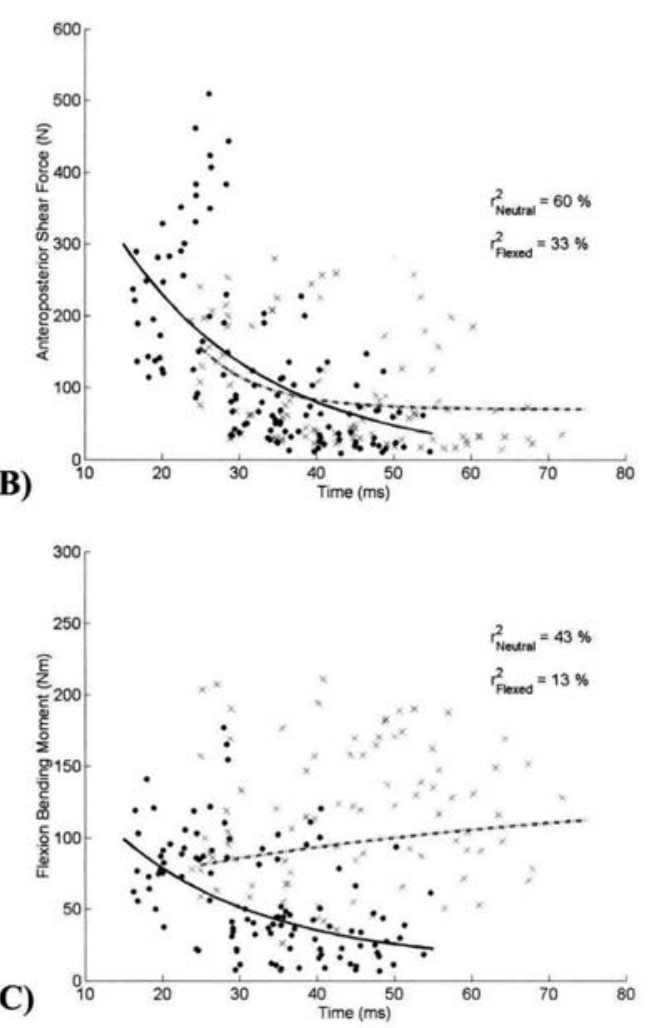

Fig 5. The experimental data and optimized curves of $(A)$ the axial reaction force, $(B)$ anterior reaction force, and $(C)$ flexion bending moment with respect to the impact contact time. The dotted points are data for neutral posture knee joints, while the $x$ points are data for flexed posture knee joints.

If we assume the contact time to be $15 \mathrm{~ms}$ for neutral knee joint and $25 \mathrm{~ms}$ for flexed knee joint, since $0 \mathrm{~ms}$ of contact time was physically unrealistic; according to the regression equations, the averages of axial reaction force, anterior reaction force, and flexion bending moment at initial stage reach $3,357 \mathrm{~N}, 287 \mathrm{~N}$, $100 \mathrm{Nm}$ for neutral standing knee joint, and reach 
Table 3. The input impact energy of in vitro impact testing tibiofemoral joint in literature

\begin{tabular}{lcccc}
\hline Researcher & Energy $(\mathrm{J})$ & Species & Joint & Method \\
\hline Hoshino $1987[16]$ & 17.6 & Human & Tibiofemoral & In vitro \\
\hline Atkinson $2000[17]$ & 11 & Canine & Tibiofemoral & In vitro \\
\hline Fukuda 2000[18] & 0.46 & Swine & Tibiofemoral & In vitro \\
\hline
\end{tabular}

$1,456 \mathrm{~N}, 155 \mathrm{~N}$, and $81 \mathrm{Nm}$ for flexed knee joint, respectively. This may give us the idea of the range for designing of sports footwear or equipment.

We successfully developed a testing apparatus to find the 6-D mechanical response of complex structures during impact loading. We found the flexed posture decreased the axial reaction force and anterior shear force but increased the flexion bending moment. The effect of stiffness of cushions on the mechanical response of knee joint during impact loading was significant for neutral posture knee joints but not for flexed knee joints. The founding of current study may imply: when designing a sports shoe which encounters the impact loading with knee joints at neutral posture, e.g. jumping, a softer cushion of shoe pad may be better to prevent knee injury. However, if the sports shoe is used for activities that knec joints are most in flexed posture, e.g. jogging, the shoe pad can be stiffer to increase the performance of energy efficiency[10].

\section{ACKNOWLEDGEMENT}

The study was supported by the National Science Council of Taiwan, the Republic of China through the grant number NSC 91-2320-B-002-159.

\section{REFERENCES}

1. Bobbert, M.F., M.R. Yeadon, and B.M. Nigg. Mechanical analysis of the landing phase in heeltoe running. J Biomech 1992;25:223-34.

2. Lafortune, M.A., E.M. Hennig, and M.J. Lake. Dominant role of interface over knee angle for cushioning impact loading and regulating initial leg stiffness. J Biomech 1996;29:1523-9.

3. Lafortune, M.A., M.J. Lake, and E.M. Hennig. Differential shock transmission response of the human body to impact severity and lower limb posture. J Biomech 1996;29:1531-7.

4. Lafortune, M.A., et al. Three-dimensional kinematics of the human knee during walking. $J$ Biomech 1992;25:347-57.

5. Milliron, M.J. and P.R. Cavanagh, Sagittal plane kinematics of the lower extremity during distane running., in Biomechanics od distance running, P.R. Cavanagh, Editor. 1990, Human Kinetics, Champaign.

6. Cheng, C.K., et al. A new approach of designing the tibial baseplate of total knee prostheses. Clin Biomech (Bristol, Avon) 1999;14:112-7.

7. Mitchell, A.D., A.M. Scholz, and V.G. Pursel. Total body and regional measurements of bone mineral content and bone mineral density in pigs by dual energy X-ray absorptiometry. J Anim Sci 2001;79:2594-604.

8. Henderson, R.C., et al. Pediatric reference data for dual X-ray absorptiometric measures of normal bone density in the distal femur. AJR Am J Roentgenol 2002;178:439-43.

9. Lee, Y.L., Kinetic and kinematic analysis of swine knee joint during impact and cyclic loading, in Inst Biomedical Engineering. 2002, National Taiwan University: Taipei, Taiwan.

10. McMahon, T.A., G. Valiant, and E.C. Frederick. Groucho running. J Appl Physiol 1987;62:2326-37.

11. Ihn, J.C., S.J. Kim, and I.H. Park. In vitro study of contact area and pressure distribution in the human knee after partial and total meniscectomy. Int Orthop 1993;17:214-8. 
12. Fukubayashi, T. and H. Kurosawa. The contact area and pressure distribution pattern of the knee. A study of normal and osteoarthrotic knee joints. Acta Orthop Scand 1980;51:871-9.

13. Hodge, W.A., et al. Contact pressures from an instrumented hip endoprosthesis. J Bone Joint Surg Am 1989;71:1378-86.

14. Kotzar, G.M., et al. Telemeterized in vivo hip joint force data: a report on two patients after total hip surgery. J Orthop Res 1991;9:621-33.
15. Torzilli, P.A., et al. Effect of impact load on articular cartilage: cell metabolism and viability, and matrix water content. J Biomech Eng 1999;121:433-41.

16. Hoshino, A. and W.A. Wallace. Impact-absorbing properties of the human knee. J Bone Joint Surg Br 1987;69:807-11.

17. Atkinson, P.J. and R.C. Haut. Injuries produced by blunt trauma to the human patellofemoral joint vary with flexion angle of the knee. J Orthop Res 2001;19:827-33.

18. Fukuda, Y., et al. Impact load transmission of the knee joint-influence of leg alignment and the role of meniscus and articular cartilage. Clin Biomech (Bristol, Avon) 2000;15:516-21. 